\title{
Philosophiques
}

\section{L’anti-psychologisme de Bradley : idéalité de la signification, jugement et universaux}

\section{Mathieu Marion}

Volume 36, numéro 1, printemps 2009

L'idéalisme britannique

URI : https://id.erudit.org/iderudit/038010ar

DOI : https://doi.org/10.7202/038010ar

Aller au sommaire du numéro

\section{Éditeur(s)}

Société de philosophie du Québec

ISSN

0316-2923 (imprimé)

1492-1391 (numérique)

Découvrir la revue

Citer cet article

Marion, M. (2009). L'anti-psychologisme de Bradley : idéalité de la signification, jugement et universaux. Philosophiques, 36(1), 53-82.

https://doi.org/10.7202/038010ar
Résumé de l'article

L'opinion est souvent exprimée que Bradley fut un des tout premiers critiques du psychologisme. Dans cet article, j'examine cette thèse en me penchant principalement sur ses Principles of Logic (1883). Je définis le psychologisme au sens étroit comme une thèse portant sur les fondements de la logique, et le psychologisme au sens large comme une thèse plus générale en théorie de la connaissance pour montrer que Bradley a rejeté les deux, même s'il n'avait pas grand chose à dire sur la version étroite. Sa critique de l'autre version est basée sur une distinction entre contenu psychologique et contenu logique, et sur sa défense de la thèse de l'idéalité du contenu logique, avant Frege et Husserl. Cependant, il tient encore à l'idée que le contenu logique provient de la perception. Après une brève présentation de ses critiques de la psychologie associationniste, je montre qu'il fait face à de véritables difficultés en essayant d'éviter de retomber dans le psychologisme en faisant appel à la distinction entre universel abstrait et universel concret. Je termine avec quelques remarques sur la place de Bradley dans l'histoire de la psychologie britannique. 


\title{
L'anti-psychologisme de Bradley: idéalité de la signification, jugement et universaux
}

\author{
MATHIEU MARION \\ Université du Québec à Montréal
}

\begin{abstract}
RÉSUMÉ. - L'opinion est souvent exprimée que Bradley fut un des tout premiers critiques du psychologisme. Dans cet article, j'examine cette thèse en me penchant principalement sur ses Principles of Logic (1883). Je définis le psychologisme au sens étroit comme une thèse portant sur les fondements de la logique, et le psychologisme au sens large comme une thèse plus générale en théorie de la connaissance pour montrer que Bradley a rejeté les deux, même s'il n'avait pas grand chose à dire sur la version étroite. Sa critique de l'autre version est basée sur une distinction entre contenu psychologique et contenu logique, et sur sa défense de la thèse de l'idéalité du contenu logique, avant Frege et Husserl. Cependant, il tient encore à l'idée que le contenu logique provient de la perception. Après une brève présentation de ses critiques de la psychologie associationniste, je montre qu'il fait face à de véritables difficultés en essayant d'éviter de retomber dans le psychologisme en faisant appel à la distinction entre universel abstrait et universel concret. Je termine avec quelques remarques sur la place de Bradley dans l'histoire de la psychologie britannique.
\end{abstract}

\begin{abstract}
One often hears the opinion voiced that Bradley was an early critique of psychologism. In this paper, I investigate that claim, focussing on his Principles of Logic (1883). I define psychologism in the narrow sense as a thesis pertaining to the foundations of logic, and psychologism in the wide sense as a more general thesis concerning the theory of knowledge, and show that Bradley rejected both, although he had little to say on the narrow version. His criticism of the wider version is based on his distinguishing between psychological and logical content and on his defence of the ideality of logical content, before Frege and Husserl. Nevertheless, he still hung to the idea that the latter harks back to ordinary perception. I then review briefly his criticisms of associationism in psychology, to show that he faced some difficulties in trying to avoid lapsing back into psychologism, with an appeal to a distinction between abstract and concrete universals. I conclude with some remarks on the palace of Bradley in the history of British psychology.
\end{abstract}

Francis Herbert Bradley, chef de file de l'idéalisme britannique, était-il un des tout premiers, sinon le premier à fournir une critique du psychologisme? C'est ce qu'affirme R. G. Collingwood dès les années trente dans un texte posthume qui ne fut publié pour la première fois qu'en 2005 :

Il n'existe pas de chose telle qu'une simple apparence. Le réel apparaît; les apparences sont apparences de la réalité. Cela contredit nettement toute la philosophie subjectiviste ou phénoméniste, de Locke à Mansel et Spencer. [...] [A]ussi simple que puisse paraitre ce résultat, une fois atteint, il a clos une époque. En mettant en avant cette thèse en apparence si évidente, Bradley a 
rendu impossible pour tout futur philosophe de substituer la psychologie à la métaphysique, comme tout philosophe, du moins en Grande-Bretagne, l'a fait depuis le milieu du dix-septième siècle (Collingwood, 2005, 243-244).

Après la guerre, Gilbert Ryle et Richard Wollheim, qu'on ne peut pas soupçonner, comme pour Collingwood, de sympathie pour Bradley, prirent le relais:

[Frege et Bradley] étaient en révolte contre le «psychologisme », c'est-à-dire en révolte contre un des éléments principaux de l'enseignement de John Stuart Mill. Mill, transmettant le legs de Hume, a cherché la solution des problèmes de logique et de théorie de la connaissance dans la psychologie associationiste. Frege et Bradley, de manière différente, distinguèrent radicalement la psychologie, d'un côté, de la philosophie et de la logique de l'autre; entre les idées, les impressions, et les sensations qui étaient le sujet de la psychologie, et tout ce qui peut bien être le sujet de la philosophie et de la logique. Ils ont tous deux détecté par la suite la même superstition philosophique se cachant derrière l'explication associationniste des pensées, c'est-à-dire la supposition que n'importe quelle pensée (ou jugement, ou proposition) est une concaténation de morceaux ayant une existence indépendante et pouvant être inspectés de manière indépendante [...]. En dernier lieu, la notion de signification devint chez Frege et Bradley ce qu'elle est restée depuis, l'instrument indispensable, si ce n'est réfractaire, du discours philosophique (Ryle, 1956, 6-7).

Cette théorie de l'esprit que j'ai attribuée aux empiristes, et plus encore leur conception concomitante de la philosophie et de la logique, Bradley l'a totalement rejetée: "En Angleterre, écrit-il, nous avons vécu trop longtemps avec une attitude psychologique.» Le rejet du psychologisme par Bradley est de la plus haute importance [...], c'est un des liens, un des rares liens qui le lie aux plus éminents et plus avant-gardistes parmi les philosophes qui furent ses contemporains. Un parallèle peut être tissé entre ses critiques sévères de l'état de la logique britannique à son époque et, par exemple, ce qui a été dit, tout à fait indépendamment mais exactement au même moment, par Gottlob Frege (Wollheim, 1959, 25-26).

Pourtant, dans «The Nature of Judgment» de 1899, G. E. Moore accusait Bradley de "psychologisme ", sans employer le terme, qui n'était pas en usage cette époque (ni à celle de Collingwood), parce qu'il soutenait que la signification (logique) d'un signe est le produit d'une abstraction à partir de son contenu (psychologique) total (Moore, 1985a, 48). On peut cependant montrer aisément que Moore n'avait pas compris Bradley ${ }^{1}$, mais je reviendrai sur cette question un peu avant ma conclusion, car je crois que cette critique masque en fait une certaine continuité entre les vues de Moore (et de Russell) et celles de Bradley.

1. En effet, pour Bradley la signification ne peut pas être conçue en ces termes, pour la simple et bonne raison que deux signes peuvent avoir la même signification. Voir Baldwin, 1990, 13-15 pour une critique détaillée des objections de Moore. 
Bradley critique du psychologisme? Cela pourrait bien être possible. En effet, dans son System of Logic, John Stuart Mill réduisait la logique à une branche de la psychologie, allant même jusqu'à remettre en cause la conception traditionnelle de l'inférence, et c'est précisément à l'encontre de cette conception "psychologiste» que Bradley écrivit ses Principles of Logic, publiés en 1883 (Bradley, 1922) ${ }^{2}$. S'il est avéré que Bradley critiqua cette forme de psychologisme dès 1883 , cela ferait bien de lui le premier critique du psychologisme en Grande-Bretagne, terre d'origine de la psychologie (dans sa version associationniste), mais aussi un précurseur des grandes figures de l'anti-psychologisme, Frege et Husserl, dont on peut faire remonter la critique du psychologisme, respectivement, aux Grundlagen der Arithmetik (Frege, 1969) et aux prolégomènes aux Logische Untersuchungen (Husserl, 1969a) ${ }^{3}$. Il serait par ailleurs difficile de soutenir que l'anti-psychologisme serait lié chez Moore ou Russell à leur rejet de l'idéalisme, qui date de 1898 (Russell, 1961, 67) - donc un an avant l'article de Moore, «The Nature of Judgment ». En effet, ni un ni l'autre n'avait encore lu Frege, et les prolégomènes aux Logische Untersuchungen n'étaient pas encore parus. La question mérite donc que nous nous y arrêtions. Il faut cependant clarifier d'abord ce qu'il faut entendre par "psychologisme", ce que ne font pas les auteurs que je viens de citer. Il en résulte un certain flou sur ce qu'on entend par ce terme, qui risque d'invalider la thèse. Je me pencherai donc tout d'abord sur cette question, pour ensuite présenter certains arguments de Bradley à l'encontre du psychologisme et de la tradition de la psychologie associationniste, en me concentrant surtout sur ses Principles of Logic (Bradley, 1922) ${ }^{4}$. Nous y verrons qu'il a en effet rejeté le psychologisme mais qu'il ne s'en est pas tout à fait dépêtré, et que les présupposés de sa critique amènent leur propre lot de problèmes. En conclusion, j'évaluerai brièvement la portée historique de la critique de la psychologie associationniste par Bradley.

\section{Variétés du psychologisme}

Les passages que je viens de citer ne sont pas univoques: le psychologisme y est présenté par Collingwood comme une thèse métaphysique, mais Ryle

2. C'est la thèse principale de Allard, 2005.

3. Pour un exemple de l'étroitesse de ce point de vue, limité aux auteurs de langue allemande, voir Kusch, 1995.

4. Outre les contraintes liées à la longueur d'un article, la raison de ce choix est simple, puisque c'est dans les Principles of Logic que se retrouvent les éléments principaux de la critique du psychologisme par Bradley, soit sa thèse de l'idéalité de la signification et sa critique de l'atomisme sous-jacent à la psychologie associationniste (discutés ci-dessous, sections 2 et 3). Cependant, la métaphysique du "sentir» (feeling) de Bradley est développée surtout dans Appearance and Reality, tandis que dans Principles of Logic elle est réduite à son aspect anti-atomiste, donc, dans cet article, je ne prétends pas discuter du cœur de la pensée de Bradley. Sur sa métaphysique du «sentir », le lecteur est renvoyé aux travaux de James Bradley (Bradley 1984 et 1991-92) et W. J. Mander (Mander 1994). L'article de Bill Mander sur la logique de Bradley (Mander, 2008), a par ailleurs été d'une grande utilité dans ma lecture des Principles of Logic. 
et Wollheim semblent aussi confondre cette thèse, à travers la référence à Frege, avec une thèse portant plus spécifiquement sur les fondements de la logique. Il faut donc d'abord distinguer, comme on le fait souvent, ce qu'on pourrait appeler un «sens étroit» d'un «sens large $»^{5}$ :

Psychologisme au sens étroit: La thèse selon laquelle la logique est fondée sur la psychologie ou n'est qu'une branche de la psychologie.

Psychologisme au sens large: La thèse selon laquelle on peut et on doit fournir une explication de la signification des mots et des énoncés - ou, en termes plus traditionnels, des concepts et des jugements - et des inférences en termes de processus mentaux 6 .

Ces définitions ne sont hélas pas uniques. Carl Stumpf, un de ses plus sévères critiques, avait dès 1891 proposé une définition encore plus large du psychologisme comme «réduction de toute recherche philosophique en général, et de toute investigation épistémologique relative à la psychologie » (Stumpf, 1891, 468) ${ }^{7}$. Ainsi défini, le "psychologisme" ressemble à un analogue de "l'historicisme", où la réduction de la philosophie s'effectue vers l'histoire plutôt que vers la psychologie; un reflet, donc, de la prétention de certaines disciplines à prendre le relais de la philosophie, malmenée durant une période de dégrisement national allemand parfois appelé avec grandiloquence "l'effondrement du système hégélien ».

D'autres définitions ne semblent pas tenir la route. Pour ne prendre que deux exemples, on doit à Karl Popper une autre définition du psychologisme, censément celle de J. F. Fries, qui le réduit de façon confuse à une forme réductionniste de phénoménisme (Popper, 1973, 93); John McDowell associe quant à lui le "psychologisme» à un mélange de béhaviorisme et de théorie sur l'introspection privée de significations linguistiques, selon lequel celles-ci seraient associées à une sphère privée propre au locuteur, dont les auditeurs seraient réduits à deviner le sens des propos sur la base de leur comportement (McDowell, 1981, 225). Cette définition farfelue lui permet de faire le reproche de psychologisme à nul autre que Dummett ${ }^{8}$ !

On distingue parfois entre psychologisme «métaphysique » et psychologisme «épistémologique» (Sober, 1978, 166-167) ${ }^{9}$. Selon cette distinction, le psychologisme «épistémologique » serait défini par la thèse selon laquelle il n'y a pas lieu de justifier les lois de la logique dans la mesure où on peut simplement montrer qu'elles sont suivies en pratique par les êtres humains.

5. Cette distinction correspond à celle que l'on retrouve dans Engel, 1996, 66-69.

6. Pour un énoncé, voir, par exemple, Dummett 1978, 88.

7. Cité dans Fisette, 1998, 231.

8. Comme le montre bien la liste établie dans Kusch, 1995, 7, l'accusation de «psychologisme " a été servie à tort et à travers au $\mathrm{xx}^{\mathrm{e}}$ siècle.

9. Ce n'est pas là la seule autre distinction dans la littérature. Voir, par exemple, Macnamara, 1986 et Cussins, 1987. 
Des «psychologistes» de la première heure tels que Friedrich Eduard Beneke ${ }^{10}$, dont Hegel, qui ne semblait pas le porter dans son cœur, avait obtenu de faire interdire les cours à Berlin ${ }^{11}$, considéraient que les lois de la logique sont vraies parce qu'elles sont nécessairement utilisées par tout être pensant; la description psychologique et la justification philosophique de ces lois ne seraient donc qu'un seul et même problème ${ }^{12}$. Cette forme de psychologisme se distingue donc par son rejet de la possibilité même d'une justification des lois de la logique, tout comme ce sera le cas, sur la base d'arguments tout autres, pour Quine dans son article célèbre sur l'épistémologie "naturalisée» (Quine, 1977). Les deux thèses énoncées ci-dessus sont par contraste "métaphysiques ", puisqu'elles soutiennent que la logique est une discipline qui traite de nos processus mentaux. C'est uniquement de ce psychologisme «métaphysique» qu'il sera question dans ce qui suit, car c'est plus spécifiquement contre celui-ci, au sens étroit comme au sens large, que furent dirigés les arguments de Bradley, de Frege et de Husserl, qui seront abordés ici.

Le psychologisme au sens étroit porte plus spécifiquement sur les fondements de la logique. Étant donné que la logique est la théorie des inférences valides, cette thèse implique donc que l'inférence doit être expliquée en termes de processus mentaux. En ce sens, le psychologisme au sens étroit est intimement relié, voire englobé dans le psychologisme au sens large. En effet, la plupart des "psychologistes » soutiennent les deux thèses de concert, et ce d'autant plus que les notions de "concept », de "jugement» et «d'inférence» formaient le cœur des nombreuses introductions à la logique publiées au XIX ${ }^{\mathrm{e}}$ siècle en Allemagne et en Grande-Bretagne ${ }^{13}$.

Le psychologisme au sens étroit est une thèse que l'on attribue très souvent à Mill, depuis Husserl, qui cite dans ses Logische Untersuchungen (Husserl 1969a, $\mathbb{S} 17$ ) le passage suivant de An Examination of Sir William Hamilton's Philosophy, paru en 1865 :

Je peux concevoir que la logique ne soit pas la théorie de la pensée en tant que pensée, mais, en tant que pensée valide, une théorie non pas de la pensée mais de la pensée correcte. C'est une science non pas distincte mais coordonnée avec la psychologie. Dans la mesure où elle est une science, elle est une partie ou une branche de la psychologie, différant d'elle d'un côté comme une partie diffère du tout et, de l'autre, comme un art diffère d'une science. Ses fondements théoriques sont entièrement empruntés à la psychologie. Il en résulte que les

10. Le terme "psychologismus" fut introduit par J. E. Erdmann en 1870, pour décrire la position de Beneke, voir Kusch, 2007. Sur Beneke, voir aussi Dussort, 1963, $29-31$.

11. Ceux qui connaissent le reproche de Hegel à Kant, qui n'aurait selon lui pas dépassé le simple niveau de la psychologie, ne devraient pas être surpris. Voir Hegel, 1971, vol. 4, 258.

12. Voir Sober, 1978, 166. En d'autres termes, la distinction entre «inné » et «a priori » est tenue ici pour nulle et non avenue.

13. On notera en passant que les Principles of Logic de Bradley s'ouvrent sur une discussion du jugement et qu'il reconnaît justement qu'on pourrait lui reprocher de « commencer au milieu (Bradley, 1922, 1). 
lois nécessaires de la pensée [...] appartiennent exclusivement à la psychologie (Mill, 1979, 359).

Plusieurs commentateurs refusent pourtant d'accoler l'étiquette à Mill ${ }^{14}$. Quoi qu'il en soit de cette question, qu'il ne nous appartient pas d'arbitrer ici $^{15}$, c'est très clairement ainsi qu'il fut perçu à l'époque. Il faut en effet bien prendre conscience du fait que le psychologisme (et l'anti-psychologisme) ne furent pas une exclusivité des philosophes allemands. Certes, des néo-kantiens de la première heure comme Fries et Beneke ont soutenu une forme de psychologisme avant Mill, mais en Grande-Bretagne celui-ci n'était pas le seul ni le premier. Parmi ses alliés se trouve un des pionniers de la psychologie, l'Écossais Alexander Bain, auteur de Senses and the Intellect (1855) et The Emotions and the Will $(1859)^{16}$. Son Logic: Deductive and Inductive ne paraîtra cependant qu'en 1870, où la thèse psychologiste est explicitement soutenue, lorsqu'il affirme faire appel aux lois de l'association au début de l'ouvrage (Bain, 1870, 36). Mill et Bain ont eu de concert une énorme influence en Allemagne à une époque où la psychologie s'est définitivement dépêtrée de l'idéalisme - où elle était restée, entre autres, dans les travaux de Moritz Lazarus et Hayim Steinthal ${ }^{17}$ — et s'est constituée comme discipline académique, de Fechner, Lotze et Brentano, à Helmholtz, Hering, Müller et Wundt. Ce qui explique que la réaction contre le psychologisme (au sens étroit) chez Frege et Husserl fut en partie dirigée contre une doctrine en provenance des îles britanniques.

Cette remarque a son importance, dans la mesure où elle explique pourquoi Bradley a lui-même réagi contre le psychologisme, au sens étroit et au sens large, avant les Allemands. En effet, ce psychologisme était très répandu au moment où il fit ses études à Oxford entre 1865 et 1869 . Henry Longueville Mansel, épigone d'Hamilton et représentant de l'école écossaise à Oxford, soutenait déjà explicitement en 1851 dans un ouvrage au titre révélateur, les Prolegomena Logica: An Inquiry into the Psychological Character of Logical Processes, que «la logique est subordonnée à la psychologie» (Mansel, 1860, 19), en faisant d'ailleurs référence à des auteurs français, dont Victor Cousin ${ }^{18}$. Toujours à Oxford, Thomas Fowler, plutôt sympathisant de l'école écossaise, répétait ce jugement dans ses Elements of Deductive Logic en 1867 (Fowler, 1867, 3). Lorsque Bradley a commencé ses études, une controverse faisait aussi rage entre Mill et Mansel, provoquée par la publication en 1865 de An examination of Sir William Hamilton's Philosophy, de Mill, qui attira l'année suivante une réplique de Mansel, The Philosophy

14. Voir, par exemple, Skorupski, 1998, 46-47, où la thèse que Mill aurait adhéré à une forme de psychologisme au sens large tel que défini ici est explicitement rejetée.

15. Pour une discussion récente de cette question, voir Godden, 2005.

16. Voir, respectivement Bain, 1864, 1865.

17. Pour un aperçu, voir Leary, 1980.

18. L'école écossaise entretenait à l'époque des liens étroits avec les philosophes français. Voir Arosio et Malherbe, 2007. 
of the Conditioned (Mansel, 1866). Malgré leur divergence sur le statut de la logique, que les uns concevaient uniquement comme une science théorique (Mansel), tandis que les autres la décrivaient aussi comme un "art» pratique (Mill et Bain), le psychologisme était donc de part et d'autre une idée convenue, que le jeune Bradley a voulu abattre ${ }^{19}$.

\section{Le jugement et l'idéalité de la signification}

En fait, Bradley ne visa pas la version étroite du psychologisme, mais sa version au sens large. Avant d'expliquer le sens de sa critique de cette dernière, il faut néanmoins souligner qu'il s'est prononcé sur le psychologisme au sens étroit, mais beaucoup plus tard, dans les «Terminal Essays » qu'il a ajoutés à la deuxième édition de ses Principles of Logic en 1922. Dans un de ces "Essays », il revient sur les rapports entre logique et psychologie, concluant en ces termes:

Donc, la psychologie et la logique, même si elles considèrent en partie la même matière, sont chacune forcées de n'aborder qu'un seul aspect de celle-ci, en la faussant au bout du compte. Bien sûr, ces sciences devraient s'éclairer mutuellement, mais ni l'une ni l'autre n'aborde la matière dans son intégralité, et la réduction de l'une à l'autre est impossible. [...] La logique et la psychologie, si elles doivent exister, doivent rester en principe indépendantes l'une de l'autre (Bradley, 1922, 613).

Le rejet du psychologisme au sens étroit est ici explicite; on peut se demander pourquoi il aura fallu attendre 1922, puisqu'il était implicite en 1883. La raison est simple: Bradley n'avait pas l'intention de prémunir la logique formelle contre tout empiètement provenant de la psychologie, pour la simple et bonne raison qu'il n'avait pas pour but de soutenir un de ces nouveaux systèmes de logique qui commençaient à apparaître à cette époque. Lorsque Frege publie ses Grundlagen der Arithmetik l'année suivante, en 1884 (Frege, 1969), il y critique le psychologisme au sens étroit dans les premières pages dans le but précis de justifier sa Begriffsschrift de 1879 ainsi que sa proposition de réduire l'arithmétique à cette dernière. Husserl n'a pas contribué à la logique formelle, mais sa critique du psychologisme dans les prolégomènes aux Logische Untersuchungen avait aussi pour but de libérer le terrain propre de la logique formelle de tout empiètement en provenance de la psychologie.

Frege et Husserl ont fourni des arguments qui visent directement le psychologisme au sens étroit, par exemple l'argument selon lequel les mathématiques et la logique possèdent le plus haut degré de précision scientifique et que ce n'est pas le cas pour la psychologie, celle-ci ne peut donc pas servir de fondement aux mathématiques et à la logique (Frege, 1969, 118-119)

19. Bien sûr, il n'était pas question de citer tout le monde, mais parmi les défenseurs d'une forme de psychologisme laissés de côté il faut mentionner la figure de Herbert Spencer, très influent à cette époque. 
(Husserl, 1969a, 68-69), ou encore que le psychologisme (au sens étroit) mène au relativisme - c'est-à-dire à la thèse selon laquelle les lois de la logique et des mathématiques varient selon les cultures ou sont spécifiques à l'espèce humaine —, et qu'il est donc contradictoire (Frege, 1969, 119) (Husserl, 1969a, 129sq.). Frege a en outre un argument selon lequel la psychologie ne peut traiter que du contexte de la découverte mais non de la justification d'une vérité mathématique ou logique (Frege, 1969, 119) ${ }^{20}$. Or, comme je le disais, Bradley ne s'était pas donné pour but de défendre la nouvelle logique formelle contre le psychologisme au sens étroit, et il n'a pas fourni d'arguments spécifiques du type de ceux que je viens de mentionner, encore moins d'arguments originaux. Il ne connaît pas les travaux de Frege et il ne fera, semble-t-il, jamais l'effort de lire ceux de son détracteur, Russell.

Tout au plus Bradley connaît-il la logique «équationnelle» exposée par W. S. Jevons dans le premier volume des Principles of Science (Jevons, 1874), et qu'il critique dans un des chapitres des Principles of Logic (Bradley, 1922, 370-388). Certaines des critiques de Bradley sont mesurées et intéressantes. Ainsi, Bradley préfigure en partie l'argument bien connu de la chambre chinoise de Searle ${ }^{21}$ lorsqu'il critique la «machine logique» de Jevons, en disant qu'elle ne peut pas fonctionner sans le secours d'un "opérateur» (Bradley, 1922, 384). D'autres critiques sont moins convaincantes, sans pour autant être dénuées d'intérêt; ainsi, par exemple, tout en admettant que le système de Jevons "prouve par ses résultats qu'il a une emprise sur la réalité » (Bradley, 1922, 370), Bradley soutient qu'inférence et substitution — la substitution étant la méthode du calcul équationnel — ne sont pas la même opération: Jevons n'épuise donc pas ce qu'on entend par inférence. Mais le fond de la critique de Jevons est le rejet par Bradley du jugement d'identité:

$$
A=A
$$

qu'il appelle «simple identité » (Bradley, 1922, 373-374) ou "identité abstraite » (Bradley, 1922, 146) et qu'il considère, à l'instar de Hegel, comme

20. Les arguments de Frege et de Husserl, dont ceux-ci ne sont pas une liste exhaustive, ont fait l'objet d'une abondante littérature secondaire; voir par exemple, Kusch, 1995; Engel, 1996; et les articles par Engel et Fisette dans Marion et Voizard, 1998. Une question importante concerne le rôle du caractère "normatif» de la logique dans l'argumentaire anti-psychologiste de Frege et de Husserl. Husserl avait critiqué les arguments des néo-kantiens (lesquels furent après tout les premiers anti-psychologistes), qui étaient fondés sur la normativité de la logique: pour cette raison, la logique ne pourrait être fondée sur des lois psychologiques qui sont contingentes et non nécessaires; ce serait une forme de sophisme naturaliste, c'est-à-dire la dérivation d'un «ought» à partir d'un «is». Voir Husserl 1969a, chapitres II et III, en particulier $\$ 19$. Je m'accorde avec Philipse, 1987, ou Kusch, 1995, 60, lorsqu'ils affirment n'y a pas de différence entre les vues de Husserl et de Frege sur le fait que la logique est fondamentalement descriptive. Pour le point de vue contraire, voir Engel, 1996, 37, 60 et 1998, 213.

21. Voir Searle, 1984, chapitre 2. 
une simple tautologie vide (Bradley, 1922, 141)22. Pour Bradley, on ne parvient donc pas à formuler quelque jugement que ce soit en assertant une «simple identité » de ce genre.

Ce genre de thèse pourrait laisser croire à une aversion de Bradley envers la logique formelle, ce qui cadrerait bien avec le fait qu'on le tient en général pour un "néo-hégélien ». En fait, la position de Bradley est cependant plus mesurée. Comme je le disais, son but dans Principles of Logic est malgré tout de défendre la possibilité de l'inférence déductive contre les critiques de John Stuart Mill. Par ailleurs, l'étiquette «néo-hégélien» qui fut apposée aux idéalistes britanniques essentiellement pour des raisons politiques $^{23}$ ne peut que porter à confusion dans plusieurs cas, dont celui de Bradley. Ce point mérite une courte digression. Comme l'a souligné James Bradley, selon moi avec raison, pour la majorité des idéalistes britanniques «Hegel ne fut à peine plus qu'une ressource» (Bradley, 1979, 14). Leur idéalisme ne découle pas d'une influence de Hegel, il n'est tout simplement pas de la même nature. Le rejet par Bradley de l'identification du réel et du rationnel, souvent cité, en est une indication claire:

Cela peut provenir d'un vice dans ma métaphysique ou d'une faiblesse de la chair qui persiste à m'aveugler, mais la notion selon laquelle l'existence pourrait être identique à la compréhension paraît aussi froide et fantomatique que le plus ennuyeux matérialisme. Que la gloire de ce monde soit en fin de compte une apparence laisse le monde plus glorieux, si nous l'éprouvons comme le spectacle d'une splendeur encore plus intense; mais le voile des sensations est une déception et une tricherie s'il cache un mouvement sans couleur d'atomes, une trame spectrale d'abstractions impalpables, ou un mystérieux ballet de catégories exsangues. Bien qu'on soit tiré vers de telles conclusions, on ne peut les épouser [...]. [Nos principes] ne constituent pas plus le Tout qui commande notre dévotion qu'une dissection de lambeaux humains déchiquetés forment la beauté de cette chair chaleureuse et vivante qui charme tant nos cœurs (Bradley, 1922, 591).

Il n'y a pas non plus de dialectique, ni d'équivalent de l'Aufhebung chez Bradley. Par ailleurs, ce qui n'est en fait qu'un reproche de "néohégélianisme» masque le fait que les idéalistes britanniques ont aussi fait énormément d'emprunts à Rudolf Hermann Lotze (qui rejetait lui aussi l'identification du réel et du rationnel). Ce dernier, relégué aux oubliettes depuis une centaine d'années, a pourtant joué un rôle majeur dans l'élaboration de

22. Voir, par exemple, Hegel, 1971, vol. 3, 33sq. (J'utilise la traduction de Serge Jankélévich parce que je considère qu'elle reste, malgré ses innombrables défauts, la meilleure que nous possédions en français.)

23. Voir l'introduction à ce numéro par Gandon et Marion. 
plus d'une doctrine idéaliste britannique ${ }^{24}$. Les emprunts de Bradley à Hegel ${ }^{25}$ - nous en verrons quelques-uns - ne doivent pas cacher ceux qu'il fit à Lotze, et qui mériteraient qu'on lui appose plutôt le qualificatif de "néolotzéen ». Un de ceux-ci, tout à fait fondamental, me semble être la notion d'idéalité de la signification.

En effet, les Principles of Logic s'ouvrent sur une discussion de la notion de jugement, où Bradley cherche à distinguer entre ce qu'il appelle "l'image psychique» (psychical image) et «la signification logique» (logical signification) (Bradley 1992, 6). Bradley cherche à faire cette distinction à l'intérieur du concept d'idée (idea), hérité de l'empirisme et de la psychologie associationniste:

24. Sur Lotze et la philosophie britannique, voir Devaux, 1932. Considérant l'importance de Lotze pour la philosophie des valeurs, l'école de Bade, Frege, et de nombreux brentaniens comme Stumpf ou Husserl, on peut s'accorder sans peine avec Passmore lorsqu'il remarque qu'au XIX ${ }^{e}$ siècle peu d'auteurs ont été autant pillés (Passmore, 1968, 51). Il n'y a pas lieu de faire la démonstration de l'importance de Lotze pour le mouvement idéaliste britannique dans le cadre de cet article, mais la simple compilation des traductions anglaises de Lotze et de Hegel montre qu'il y avait à cette époque un engouement pour Lotze, dont pratiquement toute l'œuvre a été traduite avant les années 1890, tandis qu'on ne disposait de Hegel avant 1892 que du condensé de la Science de la logique dans le premier volume de l'Encyclopédie:

1874 - G. W. F. Hegel, The Logic of Hegel (traduction du volume de l'Encyclopédie) 1884 - R. H. Lotze, Logic, in Three Books: of Thought, of Investigation, and of Knowledge - R. H. Lotze, Metaphysics, in Three Books: Ontology, Cosmology, and Psychology

- R. H. Lotze, Outlines of Metaphysic (ces Outlines sont des notes de cours)

- R. H. Lotze, Outlines of Philosophy

1885 - R. H. Lotze, Microcosmus: An Essay Concerning Man and His Relation to the World - R. H. Lotze, Outlines of Pratical Philosophy

1886 - G. W. F. Hegel, The Philosophy of Art (traduction des Cours d'esthétique)

- R. H. Lotze, Outlines of Aesthetics

- R. H. Lotze, Outlines of Psychology

1887 - R. H. Lotze, Outlines of Logic and of Encyclopedia of Philosophy

1892 - R. H. Lotze, Outlines of a Philosophy of Religion

1892 - G. W. F. Hegel, Lectures in the Philosophy of History

1894 - G. W. F. Hegel, The Philosophy of Mind (traduction du volume de l'Encyclopédie)

1895 - G. W. F. Hegel, The Philosophy of Religion

1896 - G. W. F. Hegel, The History of Philosophy

- G. W. F. Hegel, The Philosophy of Right

1910 - G. W. F. Hegel, The Phaenomenology of Spirit

1929 - G. W. F. Hegel, The Science of Logic

Bien sûr, de nombreux Britanniques lisaient Hegel dans le texte (c'est clairement le cas de Bradley), mais ces traductions indiquent bien un besoin en soi. En fait, T. H. Green avait initié la traduction de ses œuvres principales quelque temps avant sa mort en 1882, car il espérait y trouver une solution de rechange à Hegel.

25. Ceux-ci surgissent ça et là, sans parfois que Hegel soit mentionné, comme par exemple dans le reproche de Bradley à l'endroit de "l'école de l'expérience », de faire reposer sa conception de la réalité sur des séquences spatio-temporelles, l'idée que celles-ci puissent être complétées étant à ses yeux «le phantasme bien connu du mauvais infini » (Bradley, 1922, 71). Ce «spurious infinite» est une allusion au «schlechte Unendlichkeit» de Hegel, en particulier dans l'usage qui en est fait dans Hegel, 1971, vol. 4, 381. 
L'ambiguïté de la notion d' "idée " peut être dévoilée comme suit. Thèse, d'un côté, aucune idée possible ne peut être ce qu'elle signifie. Antithèse, de l'autre, aucune idée ne peut être que ce qu'elle signifie. Dans la thèse, l'idée est l'image psychique; dans l'antithèse, l'idée est la signification logique. [...] Dans ce qui suit, j'utiliserai «idée » seulement dans le sens de signification (Bradley, 1922, 6-7).

(Pour éviter toute confusion à mon tour, je réserverai dans ce qui suit le terme "idée" pour le concept de la psychologie associationniste et j'éviterai de parler comme Bradley «d'idée logique» ou simplement «d'idée » pour parler de la «signification logique».) On retrouve une distinction tout à fait analogue au $\$ 316$ de la Logik de Lotze, entre l'événement psychique momentané et son contenu, dont on peut dire qu'il est "valide » ou "qu'il vaut» (Lotze 2006, 14-15). (On notera par contre que Bradley n'utilise pas l'expression "validité » dans ses Principles.)

Pour défendre sa distinction, Bradley fait appel a des exemples plutôt imagés, comme ici le langage des fleurs, typiquement victorien, où la fleur tient lieu de l'image psychique:

Toute fleur existe et possède ses qualités propres, mais elles n'ont pas toutes une signification. Certaines ne signifient rien, tandis que d'autres tiennent en général lieu de l'espèce qu'elles représentent, tandis que d'autres encore nous rappellent l'espoir ou l'amour. Mais la fleur ne peut jamais être elle-même ce qu'elle signifie (Bradley, 1922, 3).

Ou encore cette référence biblique à Jonas :

En premier lieu, il est évident que l'idée que nous utilisons comme prédicat dans un jugement n'est pas mon état mental comme tel. «La baleine est un mammifère» ne qualifie pas les baleines réelles par mon image-mammifère. Car cette dernière m'appartient, elle est un événement dans mon histoire et, à moins que je sois Jonas, elle ne peut entrer dans une baleine réelle (Bradley, 1922, 8).

La «signification logique» est une partie du contenu, la seule qui importe pour l'analyse du jugement. Ainsi, on peut dire lorsque j'ai en tête le concept "cheval» que la signification de celui-ci est incluse dans un "ramassis de données sensorielles, d'émotions, et de sensations qui constituent mon état momentané » (Bradley, 1922, 6), mais pour la logique la situation est différente:

Cette connexion d'attributs que nous reconnaissons comme cheval est une partie du contenu de l'unique image-cheval, et ce fragment de l'événement psychique est tout ce que nous connaissons ou tout ce qui nous préoccupe en logique. L'utilisant, on traite le reste comme une enveloppe et un déchet qui n'a aucune importance pour nous et ne fait aucune différence (Bradley, 1922, 6).

Cette signification logique est pour Bradley un universel, nous reviendrons là-dessus (Bradley, 1922, 10). Il suffit d'ajouter ici que la signification logique est toujours un "signe", donc qu'elle renvoie toujours à quelque chose (Bradley, 1922, 2), et l'idée que l'image psychique ne peut pas être par 
elle-même le contenu du jugement, pour retrouver la définition du jugement par Bradley:

Le jugement est l'acte qui réfère un contenu idéel (reconnu comme tel) à une réalité au-delà de l'acte. [...] Dans l'acte d'assertion on transfère et on unit cet adjectif avec une réalité substantielle. Et on perçoit du même coup que la relation mise en avant n'est pas créée par l'acte et ne tient pas à l'intérieur ou en vertu de celui-ci, mais qu'elle est bien réelle, à la fois indépendante et au-delà de l'acte (Bradley, 1922, 10).

On remarquera par ailleurs que cette définition n'a rien de particulièrement "idéaliste». En tout cas, dans la mesure où aucun intermédiaire épistémique du genre des "sense-impressions» n'est requis, elle nie les prémisses phénoménistes dont pourrait se servir un «idéaliste subjectif» du genre de Berkeley, et un réaliste "direct", dans la tradition de Reid, pourrait à la rigueur l'adopter. Depuis l'article de G. E. Moore, "The Refutation of Idealism ${ }^{26}$, on a souvent confondu la position de Bradley avec un idéalisme subjectif comme celui de Berkeley, pour ensuite la rejeter mais Moore s'est manifestement trompé de cible. L'idéalisme de Bradley est à chercher ailleurs.

Peut-être est-ce utile d'ajouter ici que la forme du jugement n'est pas pour Bradley le traditionnel "[Sujet] est [Prédicat]». Il affirme par exemple que l'idée que la copule soit nécessaire est une "pure superstition " (Bradley, 1922, 50) et donne des contre-exemples à la forme traditionnelle, tels que l'ellipse «Feu!» (Bradley, 1922, 56-57) $)^{27}$. En fait, pour Bradley, un jugement «n'est pas une synthèse d'idées mais la référence d'un contenu idéal à la réalité » (Bradley, 1922, 56). Ce qui veut dire que tout jugement aurait pour sujet la réalité prise comme un tout, à laquelle un contenu est attribué. Donc, plutôt que de dire d'un S qu'il est $\mathrm{P}$, un jugement est plutôt de la forme «la réalité est telle que $S$ est $\mathrm{P}$ », ce qu'il écrit (Bradley, 1922, 630) comme suit, avec « $\mathrm{R}$ » pour réalité:

$$
\mathrm{S}(\mathrm{R})-\mathrm{P}
$$

Ces remarques appellent une digression, sous la forme de deux commentaires. Premièrement, on voit bien ici la place que Bradley accorde à l'unité du jugement: "Ce n'est pas vrai que le jugement contient deux idées. On peut dire au contraire qu'il n'en a qu'une» (Bradley, 1922, 10). C'est sur ce point que portera sa controverse avec Russell, où la proposition de Bradley se résume à l'idée qu'on ne peut pas analyser le contenu du jugement en des

26. Pour la traduction française, voir Armengaud, 1985, 65-86.

27. Il va sans dire que je ne cherche pas à évaluer cet argument. 
atomes indépendants pour ensuite le recomposer ${ }^{28}$. Deuxièmement, c'est aussi ici que l'idéalisme s'insère, lorsque Bradley affirmera dans Appearance and Reality que la réalité est "expérience " (Bradley, 1897, 463, 475 et 489) et qu'une telle expérience ne peut être "en dehors de l'esprit» (Bradley, 1897, 489). Cette dernière thèse réclame l'appui d'arguments qui lui sont propres, dont celui, plutôt célèbre, que l'on retrouve au chapitre XIV de Appearance and Reality:

Trouvez n'importe quel morceau d'existence, prenez n'importe quoi, qui pourrait par quiconque se faire appeler un fait, ou dont on pourrait de quelque manière que ce soit asserter l'existence, et jugez alors s'il ne consiste pas en une expérience sensible [sentient experience]. Essayez de découvrir comment vous pourriez continuer à en parler, lorsque toute perception et tout sentir [feeling] ont été éliminés, ou de pointer du doigt n'importe quel fragment, n'importe quel aspect, qui ne soit pas dérivé et qui ne soit pas toujours relatif à cette source. [...] [J]e ne peux moi-même concevoir rien d'autre que ce que ce qui est l'objet d'une expérience (Bradley, 1897, 127-128).

Et l'idée d'un fait qui ne pourrait en principe être l'objet d'une expérience ne lui semble être

[...] qu'une simple parole vide, un échec, ou une tentative de s'auto-contredire. C'est une abstraction vicieuse dont l'existence est une absurdité dénuée de sens, donc impossible (Bradley, 1897, 128).

Contrairement à ce que disent plusieurs commentateurs ${ }^{29}$, cet argument n'est pas une reprise de l'argument de Berkeley selon lequel il n'est pas possible de concevoir un objet indépendant de l'esprit sans avoir à le concevoir comme étant indépendant du fait qu'il est conçu, ce qui est une contradiction (Berkeley, 1985, 330). De l'argument de Bradley il ne faut retenir, dans le cadre de cet article, que la prémisse - dont on a envie de dire qu'elle est typiquement britannique -, soit que le seul accès que nous avons à la réalité est la perception ${ }^{30}$. Cette thèse, qui a presque le statut d'un axiome pour la pensée de Bradley, se retrouve déjà dans les Principles of Logic, où elle joue un rôle important, comme nous allons le voir:

28. Il n'est pas possible d'aborder cette controverse, et je me contenterai simplement de pointer du doigt qu'on a longtemps tenu Russell comme vainqueur parce que le point de vue de Bradley doit être erroné, or, comme Donald Davidson l'a remarqué récemment, on n'a guère avancé sur ce sujet depuis, tandis que, selon lui, Bradley a gagné son débat contre Russell (qui n'avait en fait aucun argument solide mais qui était du bon côté. Davidson, 2005, 106). Voir Candlish, 2006.

29. Voir, par exemple, Wollheim, 1959, 201. Pour une discussion plus adéquate de l'argument de Bradley, voir Mander, 1994, 128-132.

30. Lorsqu'on sait que l'œuvre de Russell a essentiellement consisté en de multiples tentatives pour montrer comment il peut dépasser les limites de sa «biographie » pour reconnaître l'existence indépendante du monde extérieur et d'autrui — les «other minds» —, on mesure la profondeur de l'influence de Bradley sur sa pensée. Pour une interprétation de la théorie de la connaissance de Russell dans ces grandes lignes, voir Marion, 2009. 
Tout jugement attribue son contenu idéel comme prédicat du réel tel qu'il apparaît dans la présentation (Bradley, 1922, 50) ${ }^{31}$.

Le réel transcende donc la présentation et nous invite à le suivre au-delà de ce qui est donné. D'un autre côté, il semble que nous n'ayons de contact avec la réalité et que nous ne touchions, pour ainsi dire, le sol nulle part en dehors de ce qui est présenté (Bradley, 1922, 72).

J'aimerais simplement conclure cette digression en insistant sur le fait, qui est important dans le cadre de cet article, que la définition du jugement de Bradley est en grande partie autonome et indifférente à l'égard de la controverse métaphysique entre idéalisme et réalisme.

J'aimerais maintenant revenir sur la distinction faite par Bradley entre image psychique et signification logique. On peut selon moi reconnaître qu'il y a dans ces premières pages des Principles of Logic une distinction qui ressemble fortement à celle qu'établira Husserl en 1896 - donc treize ans après Bradley — dans son compte rendu du livre de Kasimierz Twardowski, Sur la théorie du contenu et l'objet des représentations (Twardowski, 1993), entre «contenu psychologique » et «contenu logique» (Husserl, 1993, 350, note 1$)^{32}$. Le thème de l'idéalité de la signification trouve pour sa part son origine dans le chapitre "Le monde des idées ", dans la troisième partie de la Logik de Lotze (Lotze, 1874) ${ }^{33}$, chapitre que Bradley a très certainement $\mathrm{lu}$, et dont on peut retrouver la trace ${ }^{34}$. On retrouve en effet le contraste entre le caractère momentané de l'état psychologique et la permanence de la signification logique:

Lorsque je parle d'une idée qui reste la même à travers les changements, je ne parle pas de l'événement psychique qui est dans un flux incessant, mais d'une portion du contenu que l'esprit a fixé, et qui n'est en aucun cas un événement se déroulant dans le temps. Je parle de la signification [...] de l'or que contient le minerai, et non d'une série momentanée de notes transitoires. [...] L'événement mental est unique et particulier, mais la signification est coupée de l'existence et du reste du contenu fluctuant. [...] Elle tient lieu d'adjectif à référer à un sujet quelconque, mais qui est indifférent à tout sujet particulier (Bradley, 1922, 6).

Ce contraste est une des caractéristiques les plus importantes de l'idéalité de la signification. En effet, comme l'a montré Gottfried Gabriel (Gabriel, 2002, 42), elle permet à Lotze de faire implicitement un argument transcendantal:

31. Bradley utilise ici le terme "presentation", qui fut introduit dans Ward, 1886, pour traduire l'allemand "Vorstellung", la traduction par "représentation » risquant de porter à confusion, je traduis par "présentation». (Il suffit de songer ici aux treize sens de l'expression "Vorstellung" que distingue Husserl dans la cinquième des Logische Untersuchungen (Husserl, 1972, 315-322).)

32. Sur cette question, voir Simons 1992, 26-27 et Fisette, 1994, 30-32.

33. Ce chapitre est traduit en français dans Lotze, 2006.

34. En effet, Bradley réfère au moins à un endroit au concept de Geltung (validité) de Lotze pour lequel ce chapitre est le locus classicus; voir Bradley, 1897, 503, note 1. 
le fait que le porteur de la valeur de vérite ${ }^{35}$ - en anglais: the bearer of the truth-value - soit unique est une condition de possibilité de tout usage des valeurs de vérité, on ne peut pas parler de "vrai » et de «faux» autrement.

Une des idées principales du chapitre de Lotze sur «Le monde des idées » est que ce contenu identique n'a par ailleurs pas besoin d'être "réifié» en une sorte d' «idée » platonicienne. On ne retrouve pas non plus de trace du "platonisme" de la signification chez Bradley. En effet, pour Bradley, une signification logique

[...] ne peut jamais être un événement, avec une place dans les séquences du temps et de l'espace. Elle ne peut pas être un fait, dans nos têtes comme à l'extérieur. Et si vous isolez cette simple [signification logique], c'est un adjectif dissocié, un parasite à la dérive, un esprit sans corps qui cherche à se loger dans un autre, une abstraction à partir du concret, une pure possibilité qui n'est rien par elle-même (Bradley, 1922, 7-8).

Le contenu idéel est une idée logique; la signification telle qu'elle vient d'être définie. Il est reconnu comme tel lorsque nous reconnaissons que, en lui-même, il n'est pas un fait mais un adjectif errant [wandering adjective] (Bradley, 1922, 10).

Bradley décrit encore les significations logiques comme des «ombres paradoxales et fantômes de faits» (Bradley, 1922, 8). Cette façon imagée de s'exprimer ne doit pas cacher le fait que Bradley reprend ici à son compte la réinterprétation de la théorie des idées de Platon par Lotze selon laquelle celles-ci «sont nulle part» (Lotze, 2006, 19).

Ce thème a aussi joué un rôle important dans l'anti-psychologisme de Frege et de Husserl, où l'on retrouve mot à mot l'argument de Lotze sur la nécessité de reconnaître le contenu identique (Frege, 1971, 183) (Husserl, $1969 \mathrm{~b}, 115)$. Husserl a à plusieurs reprises reconnu sa dette envers Lotze sur cette question (Husserl, 1975, 216 et 378) , $^{36}$ mais il lui reproche toutefois de ne pas avoir dépassé le niveau du... psychologisme ${ }^{37}$ ! (Husserl, 1975, 378) L'attribution de l'origine des conceptions de Frege à Lotze a fait l'objet d'une controverse (au départ entre Michael Dummett et Hans Sluga), mais il est désormais acquis que Frege avait au moins lu certaines parties de

35. On notera au passage que la notion de "valeur de vérité » (Warheitswert), popularisée en philosophie analytique par Frege, trouve son origine dans la notion de Geltung ou Gultigkeit dans Lotze, 1879 et 2006 , $\mathbb{S} 316$, et qu'on doit en fait l'expression à Wilhelm Windelband. Voir, inter alia, Gabriel, 2002, 48.

36. Il y a une référence à Lotze, certes implicite, mais tout à fait évidente au dernier alinéa du $\ 31$ de la première des Logische Untersuchungen (Husserl, 1969b, 116). Pour la discussion la plus récente des rapports entre Lotze et Husserl dans la littérature secondaire, voir Hauser, 2003. Hauser s'appuie en particulier sur un manuscrit inédit de Husserl à propos de Lotze, qui devait à l'origine être inclus dans Logische Untersuchungen.

37. Comme le montre bien la table dans Kusch, 1995, 99, à peu près tout le monde en Allemagne et dans l'empire austro-hongrois fut accusé par quelqu'un d'autre d'être "psychologiste $» . .$. 
la Logik de $\operatorname{Lotze}^{38}$, et la distinction qu'il fait lui aussi entre le «sens" (Sinn) — et par extension les "pensées» (Gedanken) — et les «représentations" (Vorstellungen), qui ressemble fortement à la distinction faite par Bradley puisqu'il relève aussi dans de nombreux passages l'inutilité du contenu psychologique dans l'analyse du jugement (Frege, 1969, 186-187; 1971, 106107 et 181-184).

La reprise de thèses de Lotze par Bradley et sa préfiguration d'autres thèses en quelque sorte centrales à la fois pour l'école brentanienne et pour la genèse de la philosophie analytique a de quoi surprendre. Cependant, comme on peut s'y attendre, Bradley n'a pas les idées aussi claires. En effet, le lecteur perspicace aura noté que la signification logique est encore liée chez Bradley au tout de l'événement mental, dont elle est un fragment. L'origine de ce fragment n'est pas encore expliquée. Dans le premier chapitre de sa Logik, Lotze liait l'objectification des contenus nécessaires à l'activité logique de la pensée au passage des impressions aux idées. Voilà qui peut expliquer le reproche de "psychologisme» que lui adresse Husserl (Husserl, 1975, 378). Bradley avait-il mieux à proposer? Comme nous l'avons vu, la perception est l'unique source de nos «idées ", mais il insiste dans la deuxième édition sur le fait qu'il a trop exagéré le rôle des images (Bradley, 1922, 38, note 8). Il ne pourra donc pas faire appel à une forme de connaissance non perceptuelle - une sorte d'intuition ou d'intellection - d'objets comme les universaux, tels que les conçoivent les réalistes, et qui ne seraient pas dans le monde physique, cette solution ${ }^{39}$ faisant par ailleurs face à des objections assez évidentes. Et l'objection de Moore mentionnée au début de cet article refait aussi surface: si la signification logique trouve son origine dans l'expérience, par quel processus est-elle dégagée, si ce n'est par l'abstraction ? S'il y a travail de la pensée, n'y a-t-il pas psychologisme? Avant d'aller plus loin, il faut au moins noter qu'il importait avant tout, dans une critique du psychologisme, qu'une distinction fût faite entre contenu psychologique et contenu logique, pour ensuite défendre la thèse de l'idéalité de ce dernier.

\section{Psychologie associationiste et les universaux abstraits et concrets}

Pour Bradley,

[l]e fait psychologique de «l'association» est bien sûr indéniable. L'explication de ce fait que l'on retrouve dans la philosophie anglaise orthodoxe est selon moi non seulement discutable, mais fausse. Et, en plus d'être fausse,

38. De façon surprenante, la preuve la plus tangible a été versée au dossier par Dummett lui-même, dans Dummett, 1991.

39. C'est la voie qu'empruntent à la fois Husserl dans la $6^{\mathrm{e}}$ des Logische Untersuchungen, et Russell dans Problems of Philosophy (Russell, 1989, chapitre IX-XI) ou Theory of Knowledge (Russell, 2002, chapitre IX). 
elle est incompatible avec une théorie adéquate du raisonnement (Bradley, 1922, 299).

Donc, si le but de Bradley dans ses Principles of Logic est de réfuter la critique de l'inférence par Mill, sa stratégie devient ambitieuse: il se donne alors pour but de prendre le mal par la racine et de critiquer la psychologie associationniste, afin de la remplacer par un fondement psychologique plus adéquat. Elle est énoncée rétrospectivement dans une des nombreuses «Additional Notes », elles aussi ajoutées à la deuxième édition de 1922:

[...] [J]'ai jugé que, si elle est fondamentalement erronée, une philosophie doit aussi avoir une base psychologique déficiente. [...] Et un des buts de ce livre était donc de montrer qu'une logique plus vraie doit présupposer une autre compréhension des faits psychiques. En d'autres termes, le jugement et l'inférence, lorsqu'ils sont interprétés correctement par la logique, doivent montrer leur nature essentielle même dans leur origine psychologique (Bradley, 1922, 515 , n. 1).

Cette remarque révèle le sens de la critique de Bradley: une mauvaise compréhension de la logique, comme chez Mill, est basée sur une mauvaise psychologie - la psychologie associationniste - et une meilleure compréhension de la logique, c'est-à-dire du jugement et de l'inférence, demande donc en retour une refondation de la psychologie. D'où le fait que Bradley consacre deux chapitres à la critique de la psychologie associationniste (Bradley, 1922, 299-347 et 502-518). Dans une autre des "Additional Notes", il s'excuse même de la longueur excessive du premier de ces chapitres par "l'état de la psychologie en Angleterre en 1883 " (Bradley, 1922, 346, n. 1). Bradley n'a par ailleurs que des mots très durs pour cette psychologie associationniste, qu'il traite de "tradition de psychologie sénile » (Bradley, 1922, 306). Dès les premières pages il annonce ses couleurs en disant qu'en Angleterre " on a vécu trop longtemps avec une attitude psychologique» (Bradley, 1922, 2). Par quelle "psychologie» Bradley espérait-il remplacer la théorie associationniste? Comme il le reconnaît à plusieurs occasions (Bradley, 1897, 472; 1914, 153 et 1922, 346, n. 1 et 515, n. 1), Bradley s'inspire de la philosophie de l'esprit de Hegel dans la troisième partie de l'Encyclopédie des sciences philosophiques ${ }^{40}$. On peut facilement le deviner, c'est ici que les difficultés liées à sa critique du psychologisme vont surgir. Avant d'en discuter, j'aimerais présenter les critiques de Bradley à l'endroit de l'associationnisme.

La psychologie associationniste est basée sur deux lois, soit la loi de contiguïté, selon laquelle les sensations, lorsqu'elles apparaissent liées étroitement, "tendent [...] vers une forme de cohésion, de telle sorte que, lorsque par la suite l'une d'elle est présente à l'esprit, les autres sont aptes à être rappelées» (Bain, 1864, 327), et la loi de similarité, selon laquelle 
les sensations présentes «font revivre ce qui leur ressemble parmi les impressions et états passés" (Bain, 1864, 457). Dans une note à son édition de Reid (Reid, 1863, vol. 2, 897), Hamilton croyait pouvoir réduire ces deux thèses à une seule, la "réintégration" (redintegration), selon laquelle le rappel d'une partie cause le rappel du tout, un peu à la manière de la madeleine de Proust. Selon cette théorie, lorsque mon esprit a associé le goût du sucré avec l'image d'un cube de sucre, par des expériences répétées dans le passé, la vue d'un nouveau cube fait revivre le goût du sucré. Il n'en suffit pas plus pour comprendre les critiques de Bradley, qui ironise:

Crédulité Primitive murmure à l'oreille de l'enfant perplexe: ce qui a eu lieu aura encore lieu. Le sucre était sucré, et le sucre sera sucré. Et Crédulité Primitive est immédiatement acceptée comme la maîtresse de notre vie (Bradley, 1922, 324).

\section{Ses critiques visent l'atomisme sous-jacent:}

Les idées qui sont rappelées selon ces lois sont des existants particuliers. Les atomes individuels sont les unités de l'association. Et je voudrais soutenir au contraire que, dans toute reproduction, ce qui est partout opérant, c'est une identité. Aucune idée particulière n'a jamais été ou ne sera jamais associée. Ce qui est associé est et doit toujours être un universel (Bradley, 1922, 304).

En des termes plus modernes, pour Bradley, un pur particulier ne peut pas être identifié à nouveau. La raison de cette impossibilité est simple: les particuliers sont momentanés, périssent, et ne peuvent pas être ravivés pour être re-identifiés :

Selon le point de vue qui m'apparaît être vrai, parler d'association entre des particuliers psychiques n'est qu'exprimer un non-sens. Ces particuliers n'ont en premier lieu aucune permanence; leur vie ne dure qu'un moment évanescent. En deuxième lieu, ils ne peuvent jamais avoir plus d'une vie, lorsqu'ils sont morts, on en a fini avec eux. Il n'y a aucun Hadès où, exilés, ils attendraient inconsolables que l'association annonce leur résurrection et leur rappel. Lorsque le fait est corporellement enfoui dans le passé, aucun miracle n'entrouvre la tombe pour tirer vers la lumière du jour une réalité trépassée, laissée intacte par les processus qui dominent dans le monde naturel (Bradley, 1922, 306).

Et, dans un passage qui préfigure en partie un argument célèbre de Wittgenstein (Wittgenstein, 2004, \$258), il ajoute:

Nous ne possédons rien qui puisse garantir la croyance qu'un fait particulier puisse survivre à son moment ou qu'il puisse, une fois passé, revivre à nouveau. Nous savons qu'il est vrai de notre expérience actuelle que la reproduction nous présente des images, mais c'est demander un miracle pour appuyer nos fausses croyances que d'asserter que celles-ci sont les originaux passés de vie à trépas. Nous ne possédons absolument aucune forme de garantie dans notre expérience pour soutenir notre confiance dans le fait que ce qui vient à l'esprit par association est le particulier tel que nous l'avions. Car un fait particulier 
est rendu particulier par un contexte élaboré et un contenu détaillé. Et ce n'est pas ce contexte ou contenu qui revient (Bradley, 1922, 306).

Ces critiques permettent à Bradley de remettre en cause les lois de contiguïté et de similarité. Cette dernière devient particulièrement absurde, puisqu'elle suppose qu'on puisse rappeler du passé un particulier et le comparer à un autre qui est présent, comme si nous avions quelque garantie que ce soit qu'il s'agisse d'un particulier semblable. Et s'il n'y pas d'association par similarité, il n'y a pas d'association du tout (Bradley, 1922, 319) ${ }^{41}$. Seule la loi de "réintégration" trouve grâce aux yeux de Bradley, quoique sous une forme différente, car pour Bradley ce sont des universaux qui sont à l'œuvre dans la "réintégration ». En effet, selon lui, on ne peut re-identifier que des universaux: "une identité de contenu dans différents contextes est et doit être un universel » (Bradley, 1922, 309), et toute nouvelle perception «re-particularise» cet universel, ce qui n'est pas la même chose qu'une «association» ou une « reproduction» (Bradley, 1922, 308-309). Donc, la "réintégration par identité » offre la meilleure explication des faits. Pour une présentation $X$ dont le contenu a pour parties $a, b$, ct et $d$, Bradley nous décrit cette "réintégration" en ces termes:

C'est une connexion non pas entre ce $a$ et ce $b$, ou ce $c$ et ce $d$, mais entre les universaux $a$ et $b$, ou $c$ et $d$. C'est une particularité de l'esprit qui se manifeste dans le fait que, étant donné que nous avons une partie du contenu qui apparaît dans $X$, ce simple universel $a, b, c$, ou $d$, lorsque donné dans un ensemble différent de particuliers, peut instituer à nouveau par son identité idéale n'importe quel des universaux $a, b, c$, et $d$, et ce malgré le fait que tout ce qui a particularisé et donné son existence à ce contenu en $X$ soit disparu. Il en fera le rappel dans des habits particuliers, mais ceux-ci seront déterminés par les circonstances mentales du moment et ne seront pas les habits de son existence passée. Et ces habits particuliers [...] ne sont pas le lien qui est opérant dans la reproduction. Ce qui est opérant est la connexion entre les universaux, et la base de cette opération est l'identité idéale d'un élément dans ce qui est présent et dans ce qui est passé (Bradley, 1922, 307) ${ }^{42}$.

Je termine là-dessus ma présentation des critiques de Bradley à l'endroit de la psychologie associationniste. On notera le fait que Bradley n'y précise pas plus sa conception des universaux que lorsqu'il définissait la signification logique d'un jugement comme étant un universel. On pourrait penser qu'il adopte la conception traditionnelle de la distinction entre universaux et particuliers et, étant donné sa position sur l'idéalité de la signification selon laquelle les universaux ne sont en soi que des "adjectifs errants » et

41. Bradley reformule par ailleurs la loi de contiguïté (Bradley, 1992, 315) de telle sorte qu'on ne peut parler non plus d'association pour cette loi.

42. Il faut noter que cette conception trouve sa source dans un autre emprunt à Hegel. En effet, celui-ci décrit dans le $\$ 456$ de l'Encyclopédie des sciences philosophiques l'association comme subsomption de particuliers sous des universaux. Voir, Hegel, 1988, 251. 
n'ont pas d'existence propre en dehors de leurs particularisations - , qu'il adopte forcément une position nominaliste "modérée » sur la question du statut ontologique des universaux ${ }^{43}$, comme l'ont fait après lui (sur des bases certes bien différentes) Stout et Cook Wilson ${ }^{44}$. Ce serait méconnaître la conception des «universaux concrets» de Bradley ${ }^{45}$, développée par la suite surtout par Bernard Bosanquet ${ }^{46}$, qui est une des idées les plus caractéristiques de l'idéalisme britannique. Cette doctrine doit être exposée succinctement à son tour.

Bradley reconnait une distinction entre particuliers et universaux mais distingue en outre leurs versions abstraites et concrètes. Selon lui, l'expérience nous livre une réalité composée d'individus; seuls ces derniers sont réels (Bradley, 1922, 45), et la réalité n'est pas composée de "connexions d'adjectifs » (Bradley, 1922, 46). Ces individus existent par eux-mêmes et ne sont pas des simples, mais des complexes. Ils perdurent dans le temps et l'espace et sont re-identifiables, donc ils ne sont pas des particuliers mais des universaux:

Nous sommes habitués à parler et à croire en des réalités qui existent à plus d'un moment du temps ou d'une portion de l'espace. Toute identité de ce genre serait une identité qui apparaît et reste la même dans la différence, et serait donc un véritable universel (Bradley, 1922, 45) ${ }^{47}$.

Ces individus sont donc des universaux concrets. Pour Bradley comme pour Bosanquet, les noms propres indiquent des universaux : Jules César est un universel concret ${ }^{48}$. Bradley argumente aussi par la suite qu'un individu, vu sous un autre angle, celui non pas de son identité dans la différence mais de son opposition à d'autres individus, est aussi un particulier concret (Bradley,

43. Voir Acton, 1936-37, 422, qui reconnaît ce point sans réaliser qu'il s'agit d'une forme de nominalisme.

44. J'emploie l'expression «nominalisme modéré » au sens où on l'entend dans Moreland, 2001, chapitre 1. Pour Stout, discuté dans Moreland, 2001, voir Stout, 1966. Pour Cook Wilson, aujourd'hui ignoré, mais dont Stout a repris plusieurs idées, voir Wilson, 1926, 670691 et 707-714.

45. Pour un exposé critique de cette doctrine, voir l'excellent article de H. B. Acton, «The Theory of Concrete Universals » (Acton, 1936-37). Sur l'origine de la notion d'universel concret chez Hegel, voir Stern, 2007.

46. De ce dernier, voir tout particulièrement Bosanquet, 1888, ainsi que les commentaires, Houang, 1954, 29sq. et Mander, 2000.

47. Bradley fait ici allusion à la doctrine de «l'identité dans la différence ", qui est un des piliers de cette théorie des universaux concrets. (L'autre étant le rejet de la doctrine traditionnelle de la variation en proportion inverse de l'extension et de l'intension (plus l'extension est grande, moins l'intension l'est); Bradley considère cette doctrine comme "fausse et frivole " (Bradley, 1922, 170); selon lui, plus l'extension est grande plus l'intension est grande.) La doctrine de "l'identité dans la différence " est basée sur le rejet de l'identité $A=A$, que nous avons vue cidessus, parce qu'elle ne réussit pas à exprimer un jugement. Pour Bosanquet, "l'identité est un universel, un point de rencontre des différences, ou une synthèse des différences et donc toujours, en un sens, concrète » (Bosanquet, 1888, 358).

48. Bosanquet, 1888,359 , note 1 , fait remonter cette thèse à Sigwart. 
1922, 188). Je suppose que les universaux concrets, en tant qu'identité dans la différence, sont donc cette identité qui fait fonctionner la "réintégration ".

Par ailleurs, lorsqu'on considère pour lui-même un aspect indissociable d'une chose sans laquelle il ne peut exister, donc séparément de cette chose, et qu'en vertu de cela on lui impute une existence séparée, nous avons alors affaire à une "abstraction ", selon Bradley qui semble viser ici la théorie de l'abstraction de l'empirisme et de la psychologie associationniste, selon laquelle l'universel «rougeur» est le produit d'une abstraction par la pensée à partir de plusieurs instances. Le particulier et l'universel abstraits sont des "créations de l'esprit ", des distinctions dans le tout de l'expérience, "solidifiées en unités» indépendantes (Bradley, 1922, 189). Ceux-ci ne peuvent donc être réels. On se rappellera que Bradley définissait la signification logique comme un universel, qui n'a pas d'existence propre (à l'opposé de ce à quoi elle réfère). Cette définition est reprise au chapitre VI:

Un universel n'est rien d'autre qu'un adjectif. C'est une épithète dissociée, une ombre qui, en dehors de son corps, n'est rien et ne peut pas exister (Bradley, 1922, 187).

Et elle est amplifiée:

L'universel abstrait et le particulier abstrait sont tous deux non réels, parce qu'ils ne sont pas des noms pour l'individuel. Ils prennent deux aspects ou caractères du tout et, les tournant en existences individuelles, assertent alors leur réalité. Mais une partie du tout ne peut tenir par elle-même seulement dans notre tête. Elle n'est qu'un adjectif, une distinction interne que l'on veut considérer comme un fait substantiel. On peut voir que cela est vrai des universaux abstraits. L'unité ou l'identité d'un homme, nous le savons, ne se trouve pas à la suite d'une recherche dans la série des phénomènes mentaux. Mais cela est aussi vrai des particuliers abstraits. Si vous prenez les atomes au sérieux et niez leur extension, vous réalisez tout de suite que vous avez affaire à quelque chose qui ne peut pas être un fait (Bradley, 1922, 188).

On peut donc résumer la doctrine en laissant la parole à Bradley:

L'universel abstrait et le particulier abstrait sont ce qui n'existe pas. Le particulier concret et l'universel concret ont tous deux une réalité et sont différents noms pour un individu (Bradley, 1922, 188).

Toute cette théorie des universaux concrets tombant à l'eau si l'on adopte le point de vue extensionnel qui est celui de la logique contemporaine, elle fait donc face à de sérieuses difficultés. Mon propos n'est cependant que de relever quelques difficultés liées à cette théorie dans le cadre de la critique par Bradley du psychologisme au sens large.

Une première difficulté a été soulevée par H. B. Acton (Acton, 1936-37, 423-424), qui note avec raison que la description des universaux abstraits par Bradley correspond à celle des significations logiques, que nous avons vue dans la section précédente. Dans les deux cas, il s'agit d'abstractions qui 
n'ont aucune existence propre lorsque prises isolément. D'après ce qu'il en dit, il est d'autre part clair que pour Bradley les signification logiques ne sont pas des créations de l'esprit mais que les universaux abstraits sont de telles créations. Doit-on en conclure que les universaux abstraits ne font pas partie de la signification logique?

Une deuxième difficulté est liée au fait que, pour Bradley, tout semble se passer comme si l'esprit pouvait dans un premier temps acquérir des contenus de signification idéaux, pour juger dans une deuxième étape. Il serait alors possible «d'avoir en tête» des contenus «sans juger» (Bradley, 1922, 26-27), donc l'acte de référer dans le jugement serait un acte bien distinct de l'abstraction par laquelle les contenus sont obtenus. Or rien de cela n'est acceptable pour Bradley, pour deux raisons, et il a changé d'avis assez rapidement après la publication des Principles. Premièrement, il y a risque de réintroduire un dualisme entre la pensée qui aurait «en tête » des contenus et la réalité à laquelle ces contenus sont renvoyés dans le jugement. Ce dualisme est incompatible avec le monisme qu'il défend ${ }^{49}$. Deuxièmement, on peut utiliser ces floating ideas pour construire une représentation systématique de la réalité qui, bien que cohérente, est fausse. C'est l'objection que Russell servira à la théorie de la vérité cohérence (Russell, 1997, 191), et contre laquelle Bradley s'était prémuni en réclamant un lien étroit entre tout contenu et la réalité ${ }^{50}$. Il corrige son tir et précise donc dans Appearance and Reality que tout contenu doit référer:

Une pensée qui ne serait seulement que "dans ma tête» ou une simple idée séparée de toute relation au monde réel est une fausse abstraction. Car nous avons vu qu'entretenir une pensée c'est, plus ou moins clairement, la référer à la Réalité. Et, par conséquent, une idée complètement libre de toute référence serait une auto-contradiction (Bradley, 1897, 350).

Dans un des «Terminal Essays» de la deuxième édition des Principles, il écrit: "Il n'y a pas et il ne peut pas y avoir une chose telle qu'une simple idée, une idée en dehors de tout jugement, se tenant et flottant par ellemême » (Bradley, 1922, 640). Dans une note elle aussi ajoutée à la deuxième édition, il va même jusqu'à dire que "tout ce qui est concevable existe en un sens» (Bradley, 1922, 195, note 27). Donc, aucun contenu, aucune signification logique ne doit être au bout du compte complètement déconnectée de son contexte d'origine. Les conceptions de Bradley l'empêchent donc d'émanciper complètement sa conception du jugement de la sphère de la psychologie, et donc de se dépêtrer complètement de tout psychologisme. Il ne nous donne en outre aucune réponse aux questions soulevées à la fin de la section précédente. Il n'explique pas non plus par la suite, puisqu'il doit bien reconnaître que les universaux abstraits sont concevables (et qu'ils ne sont

49. Pour Bradley le jugement n'est pas une relation externe et ne doit pas faire place à un «sujet»; voir Bradley, 1914, 326-327.

50. Voir Holdcroft, 1998, 171sq. 
pas des créations de l'esprit), comment ils sont insérés dans la réalité, mais ne le fera pas.

On remarquera cependant ceci : l'idée que « tout ce qui est concevable existe en un sens » est une idée que l'on retrouve, paradoxalement, dans les toutes premières théories "réalistes" de Moore et de Russell, où un "concept» (Moore) ou un «terme» (Russell) doit correspondre à chaque partie d'un jugement ${ }^{51}$, ce qui indique, encore une fois, l'importance du rôle de Bradley dans la genèse de la philosophie analytique. J'ai mentionné au début de l'article la critique de Bradley par Moore dans "The Nature of Judgment ». Selon Moore, au moment où il écrit cet article, une proposition est composée d'éléments, appelés "concepts", qui sont eux-mêmes ce sur quoi porte la proposition, et la proposition est l'objet d'un jugement, donc les «concepts» forment une partie du jugement, mais les «concepts» — et il en va de même pour les «termes » de Russell — ont une existence indépendante de l'esprit ${ }^{52}$, ce qui est censé rendre leurs théories «réalistes». Toujours selon Moore, Bradley ne reconnaissant pas d'existence indépendante aux composantes du jugement, il resterait prisonnier d'une conception que l'on pourrait appeler "psychologiste » et il serait donc contraint d'adopter une forme d'idéalisme ressemblant étrangement à celui de Berkeley, que Moore critiqua par la suite dans "The Refutation of Idealism» (Moore, 1985b). Nous avons vu qu'il n'en est rien: l'essentiel pour Bradley est la thèse que tout jugement dit quelque chose à propos de la réalité, et non à propos de contenus psychologiques (Bradley, 1922, 41). Et Bradley ne remet pas en cause l'existence d'une réalité externe au jugement; la critique de l'idéalisme dans "The Nature of Judgment" et "The Refutation of Idealism» rate donc sa cible. Par ailleurs, si Bradley ne dégage pas totalement le contenu logique de la sphère du psychologique, la solution de Moore et de Russell n'est guère plus satisfaisante. Ni Moore ni Russell n'ont songé à ce moment à une notion de sens comme celle de Frege, et il envisagèrent plutôt, pour éviter toute forme de psychologisme ou d'idéalisme, de faire entrer des parties du monde extérieur dans la composition de la proposition, ce qui donna pour résultat l'étrange théorie selon laquelle le mont Blanc et toute sa neige font partie du jugement «Le mont Blanc est d'une hauteur de plus de 4000 mètres ", que Russell exposera dans une lettre à Frege, en décembre 1904 (Frege, 1980, 169). Il n'est bien sûr pas question de discuter ces développements plus avant, j'aimerais simplement pointer du doigt la proximité des idées de Bradley.

51. En effet, pour Russell, tout ce qui peut être objet de pensée est un «terme », et « tout terme a une existence, c'est-à-dire est en un sens» (Russell, 1903, 49). Moore utilise "concept » là où Bradley dit «signification» ou «idée logique »; voir Moore, 1985a, 47. Et Russell reconnaît (Russell, 1903, 44, note *) que sa notion de «terme» est une variante de la notion de «concept» de Moore...

52. Voir, par exemple, Moore, 1985a, 50. 


\section{Conclusion: l'impact de Bradley}

Je crois qu'il est inutile de revenir sur l'influence de l'œuvre de Bradley sur la philosophie britannique, car elle est déjà bien connue. J'ai montré que son influence sur la pensée de Moore et Russell n'était en fait pas aussi nettement négative qu'ils ont bien voulu le laisser paraître, et qu'au moins sur un point ces derniers semblent avoir repris un élément important de la pensée de Bradley. J'aimerais conclure cet article sur quelques remarques concernant la critique de la psychologie associationniste par Bradley, dans le but d'en proposer une vision plus nuancée et plus positive, afin de combattre les préjugés que l'on entretient d'ordinaire à son égard.

Un survol de l'histoire de la psychologie en Grande-Bretagne ${ }^{53}$ montre qu'elle a éprouvé de graves difficultés à se faire reconnaître comme discipline universitaire indépendante, et ce malgré les origines britanniques dans l'associationnisme, de Hobbes à Hartley ${ }^{54}$. Quelques dates permettent de s'en faire une idée: le premier cours de psychologie est donné à Cambridge par James Ward en $1878^{55}$, mais on lui avait cependant refusé l'année précédente des fonds pour un laboratoire de psychophysique. Il obtiendra des fonds à partir de 1891, et le premier véritable laboratoire sera celui de W. H. R. Rivers, en 1897. James Sully est engagé à Londres en 1878 et inaugurera son laboratoire en 1898. En comparaison, le laboratoire de Wundt à Leipzig le premier dans l'histoire - est fondé en 1879. À Oxford, l'université de Bradley, la situation est catastrophique: malgré la création du Wilde Readership in Mental Philosophy, en 1897 (dont le premier détenteur sera G. F. Stout), la première chaire en psychologie ne sera fondée qu'en $1946^{56}$ ! Philosophes et psychologues étaient pourtant solidaires, comme le montre la revue Mind, fondée en 1876 par Alexander Bain et George Croom Robertson, dont le sous-titre, disparu sous la direction de Ryle, était: "A Quarterly Review of Psychology and Philosophy». On impute souvent la responsabilité de ces déboires institutionnels aux philosophes, en particulier aux idéalistes, et l'anti-psychologisme de Bradley pourrait laisser croire à une détestation de la discipline aux effets délétères. Mais les idéalistes ne peuvent guère être tenus pour seuls responsables. En effet, Bradley vivait à Oxford comme un ermite, et ce sont les « réalistes » et leurs héritiers, les "philosophes du langage ordinaire» qui dominèrent ${ }^{57}$. Ceux-ci s'opposèrent

53. Il n'y a que très peu de travaux d'histoire de la psychologie britannique. Voir Hearnshaw, 1964 et Bunn, Lovie \& Richards, 2001.

54. Sur cette dernière, l'ouvrage de Théodule Ribot (Ribot, 1914) demeure une référence malgré certaines lacunes. Voir aussi Warren, 1921.

55. On refusait, semble-t-il, de "mettre l'âme humaine sur une balance », car cela serait «insulter la religion» (Hearnshaw 1964, 171).

56. George Humphrey, qui avait fondé le Département de psychologie à l'Université Queen's, en Ontario, entrera en fonction en 1947.

57. Sur cette question, voir Marion, 2000. 
ouvertement et parfois de façon méprisante ${ }^{58}$ à la psychologie, de H. A. Prichard et H. W. B. Joseph à Gilbert Ryle ${ }^{59}$. L'œuvre de Bradley recèle au contraire de fines analyses qui sont autant de contributions à la psychologie; il suffit pour s'en rendre compte d'ouvrir les volumes de ses Collected Essays (Bradley, 1935) où l'on retrouve des textes au titre évocateur tels que "A Defence of Phenomenalism in Psychology ", "Why Do we Remember Forwards and not Backwards? " et "What Do we Mean by the Intensity of Psychical States?». Ses débats sur la notion d'attention avec James Ward, qui était lui-même idéaliste ${ }^{60}$, ou sur la notion de ressemblance avec William James, dont le concept d' "expérience pure» est une reprise de la notion d' "expérience » de Bradley ${ }^{61}$, sont aussi d'importantes contributions à cette discipline. Il ne faut donc pas confondre sa critique de la psychologie associationniste avec un rejet en bloc ou un mépris de la psychologie.

On considère la parution de l'entrée «Psychology », par James Ward, dans la $9^{\mathrm{e}}$ édition de l'Encyclopaedia Britannica, en 1886, comme un point tournant dans l'histoire de la psychologie britannique, parce que Ward y présentait pour la première fois une vision d'ensemble de la discipline qui n'était plus basée strictement sur l'associationnisme ${ }^{62}$; la riposte de Bain en dit long ${ }^{63}$. Je crois que la parution de Principles of Logic, deux ans auparavant, avec sa critique de l'associationnisme, a aussi contribué au déclin de l'école associationniste (quoique les critiques présentées ci-dessus ne constituent pas l'ensemble de l'argumentaire contre l'associationnisme, loin s'en faut). Celleci ne sera bientôt plus représentée que par James Sully et George Croom Robertson, à Londres. Ce dernier reconnaîtra le rôle fondamental des critiques de Bradley dans son entrée "Association of Ideas" pour l'Encyplopaedia Britannica (Robertson, 1910, 786 ${ }^{64}$, tout comme G. F. Stout dans son Analytic Psychology (Stout, 1896, 45-49) ${ }^{65}$. Il faut donc reconnaitre que Bradley a joué un rôle important dans la transition de la psychologie associationniste à la "psychologie descriptive ", c'est-à-dire à l'école de Brentano,

58. Je ne connais cependant pas d'attitude aussi méprisante que celle des héritiers français de Comte, au siècle dernier, telle qu'on la retrouve dans Canguilhem, 1958, et Foucault, 2001.

59. Voir, par exemple, la polémique entre Prichard, 1907, et Joseph, 1910-1911, d'un côté, et Stout, 1907, 1911 de l'autre. On notera par ailleurs que les critiques du dernier idéaliste à Oxford, Collingwood, dans An Essay on Metaphysics (Collingwood, 1998, chapitres XI-XII) s'étaient attiré une réplique dans Hearnshaw, 1942. Sur Collingwood et la psychologie, voir Connelly et Costall, 2000.

60. Voir ses Gifford Lectures: Ward, 1909 et 1911.

61. Sur les rapports entre Bradley et James, voir Sprigge, 1993.

62. Ward et Bradley était donc des alliés dans la critique de l'associationnisme, ce qui ne veut pas dire qu'ils s'accordaient sur tout. Sur leurs divergences, voir Wollheim, 1959, 133-138.

63. Voir Bain, 1886, 1887.

64. La réimpression de cette entrée dans Robertson, 1896, n'inclut cependant pas la section sur Bradley.

65. Voir aussi Hearshaw, 1964, 129-131. 
que Stout introduisit en Grande-Bretagne dans son Analytic Psychology, l'expression "psychologie analytique » étant d'ailleurs sa traduction de «deskriptive Psychologie ${ }^{66}$. À ce titre, tout autant que pour son influence sur un fondateur de la philosophie analytique comme Russell, il mérite qu'on reconnaisse l'importance historique de son œuvre, plutôt que de la concevoir, comme celle des autres idéalistes britanniques, comme une simple aberration, sans prendre la peine de la lire ${ }^{67}$.

\section{Bibliographie}

Acton, H. B. «The Theory of Concrete Universals », Mind, n. s., vol. 45, 1936, 417431 et vol. 46, 1937, 1-13.

Allard, J. The Logical Foundations of Bradley's Metaphysics. Judgement, Inference and Truth, Cambridge, Cambridge University Press, 2005.

Armengaud, F. G. E. Moore et la genèse de la philosophie analytique, Paris, Klincksieck, 1985.

Arosio, E. et M. Malherbe. Philosophie française et philosophie écossaise 17501850, Paris, Vrin, 2007.

Bain, A. The Senses and the Intellect, $2^{\mathrm{e}}$ édition, Londres, Longmans \& Green, 1864.

- The Emotions and the Will, $2^{\mathrm{e}}$ édition, Londres, Longmans \& Green, 1865.

-. Logic: Deductive and Inductive, Londres, Longmans \& Green, 1870.

—_. "Mr James Ward's 'Psychology' », Mind, vol. 11, 1886, 457-477.

—_. "On 'Association' Controversies ", Mind, vol. 12, 1887, 161-182.

Baldwin, T. G. E. Moore, Londres, Routledge, 1990.

Bell, D. "The Revolution of Moore and Russell: A Very British Coup?", dans A. O'Hear (dir.), German Philosophy since Kant, Cambridge, Cambridge University Press, 1999, 137-66.

Berkeley, G., Euvres I, Paris, Presses Universitaires de France, 1985.

Bosanquet, B. «The Philosophical Importance of a True Theory of Identity ", Mind, vol. 13, 1888, 356-369.

Bradley, F. H. Appearance and Reality, $2^{\mathrm{e}}$ édition, Oxford, Clarendon Press, 1897.

- Essays on Truth and Reality, Oxford, Clarendon Press, 1914.

- The Principles of Logic, $2^{\mathrm{e}}$ édition, Londres, Oxford University Press, 1922.

- Collected Essays, 2 volumes, Oxford, Clarendon Press, 1935.

Bradley, J. «Hegel in Britain: A Brief History of British Commentary and Attitudes», Heythrop Journal, vol. 20, 1979, 1-24 et 163-182.

- - «F. H. Bradley's Metaphysics of Feeling and its Place in the History of Philosophy », dans A. Manser et G. Stock (dir.), The Philosophy of F. H. Bradley, Oxford, Clarendon Press, 1984, 227-242.

66. Sur le rôle joué par Stout, et donc celui de l'école de Brentano dans l'émergence de la philosophie analytique chez ses étudiants Moore et Russell, voir Bell, 1999.

67. Une version antérieure de cet article a été lue au colloque «Comment être idéaliste? Bradley et Collingwood» qui a eu lieu à l'Université Blaise Pascal de Clermont-Ferrand en juin 2006. J'aimerais remercier Denis Fisette pour nos nombreuses discussions sur les questions traitées dans cet article, en particulier dans le cadre du séminaire «Philosophie et psychologie en Grande-Bretagne de 1850 à 1940 », que nous avons enseigné conjointement à l'Université du Québec à Montréal en 2009. 
—. «Relations, intelligibilité et non-contradiction dans la métaphysique du sentir de F. H. Bradley: une réinterprétation », Archives de philosophie, vol. 54, 1991, 529-551 et vol. 55, 1992, 77-91.

Bunn, G. C., A. D. Lovie et G. D. Richards (dir.) Psychology in Britain. Historical Essays and Personal Reflections, Leicester, BPS Books, 2001.

Candlish, S. The Russell/Bradley Dispute and its Significance for Twentieth Century Philosophy, Basingstoke, Palgrave MacMillan, 2006.

Canguilhem, G. "Qu'est-ce que la psychologie?", Revue de métaphysique et de morale, 1958, 12-25.

Collingwood, R. G. An Essay on Metaphysics, $2^{\mathrm{e}}$ édition, Oxford, Clarendon Press, 1998.

- An Essay on Philosophical Method, $2^{\mathrm{e}}$ édition, Oxford, Clarendon Press, 2005.

Connelly, J. et A. Costall «R. G. Collingwood and the Idea of an Historical Psychology ", Theory and Psychology, vol. 10, 2000, 147-170.

Cussins, A. "Varieties of Psychologism ", Synthese, vol. 70, 1987, 123-154.

Davidson, D. Truth and Predication, Cambridge MA, Harvard University Press, 2005.

Devaux, P. Lotze et son influence sur la philosophie anglo-saxonne. Contribution à l'étude historique et critique de la notion de valeur, Bruxelles, Lamertin, 1932.

Dummett, M. A. E. Truth and Other Enigmas, Londres, Duckworth, 1978.

—. "Frege's 'Kernsätze zur Logik' ", réimprimé dans Frege and Other Philosophers, Oxford, Clarendon Press, 1991, 65-78.

Dussort, H. L'école de Marbourg, Paris, Presses Universitaires de France, 1963.

Engel, P. Philosophie et Psychologie, Paris, Gallimard, 1996.

—. "L'anti-psychologisme est-il irrésistible? ", dans Marion et Voizard, 1998, 211-226.

Fisette, D. Lecture frégéenne de la phénoménologie, Combas, Éditions de l'Éclat, 1994.

_. "L'anti-psychologisme de la phénoménologie et la psychologie », dans Marion et Voizard, 1998, 227-259.

Foucault, M. «Philosophie et psychologie», dans Dits et écrits. Tome I, Paris, Gallimard, 466-476.

Fowler, T. The Elements of Deductive Logic, Oxford, Clarendon Press, 1867.

Frege, G. Fondements de l'arithmétique, Paris, Éditions du Seuil, 1969.

—. Écrits logiques et philosophiques, Paris, Éditions du Seuil, 1971.

- Philosophical and Mathematical Correspondence, Oxford, Blackwell, 1980.

Gabriel, G. "Frege, Lotze, and the Continental Roots of Early Analytic Philosophy », dans E. Reck (dir.), From Frege to Wittgenstein, Oxford, Oxford University Press, 2002, 39-51.

Godden, D. M. «Psychologism in the Logic of John Stuart Mill : Mill on the Subject Matter and Foundations of Ratiocinative Logic ", History and Philosophy of Logic, vol. 26, 2005, 115-143.

Hauser, K. "Lotze and Husserl ", Archiv für Geschichte der Philosophie, vol. 85, 2003, 152-178.

Hearnshaw, L. S. "A Reply to Professor Collingwood's Attack on Psychology ", Mind, n. s., vol. 51, 1942, 160-169.

-. A Short History of British Psychology, 1840-1940, Londres, Methuen, 1964. Hegel, G. W. F., 1971, Science de la logique, 4 volumes, Paris, Aubier. 
—. Encyclopédie des sciences philosophiques. III. Philosophie de l'esprit, Paris Vrin, 1988.

Holdcroft, D. «Bradley and Floating Ideas », dans G. Stock (dir.), Appearance versus Reality: New Essays on the Philosophy of F. H. Bradley, Oxford, Clarendon Press, 163-180.

Houang, F. Le néo-hégélianisme en Angleterre. La philosophie de Bernard Bosanquet 1848-1923, Paris, Vrin, 1954.

Husserl, E. Recherches logiques. Tome 1: Prolégomènes à la logique pure, Paris, Presses Universitaires de France, 1969 [1969a].

- Recherches logiques. Tome 2: Recherches pour la phénoménologie et la théorie de la connaissance. Première partie: Recherches I et II, Paris, Presses Universitaires de France, 1969 [1969b].

- Recherches logiques. Tome 2: Recherches pour la phénoménologie et la théorie de la connaissance. Deuxième partie: Recherches III, IV et V, $2^{\mathrm{e}}$ édition, Paris, Presses Universitaires de France, 1972.

—. Articles sur la logique, Paris, Presses Universitaires de France, 1975.

- «Discussion de Twardowski, Sur la théorie du contenu et de l'objet des représentations ", dans Husserl-Twardowski, Sur les objets intentionnels. 18931901, Paris, Vrin, 349-356.

Jevons, W. S. The Principles of Science. A Treatise on Logic and Scientific Method, 2 volumes, Londres, MacMillan, 1870.

Joseph, H. W. B. "The Psychological Explanation of the Development of the Perception of External Objects ", Mind, n. s., vol. 9, 1910, 305-321 et 457-469, et vol. 10, 1911, 161-180.

Kusch, M. Psychologism. A Case Study in the Sociology of Philosophical Knowledge, Londres, Routledge, 1995.

—. "Psychologism», dans E. N. Zalta (dir.) The Stanford Encyclopedia of Philosophy, 2007. En ligne: http://plato.stanford.edu/entries/psychologism/

Leary, D. E. "German Idealism and the Development of Psychology in the Nineteenth Century", Journal of the History of Philosophy, vol. 18, 1980, 299-317.

Lotze, R. H. Logik, drei Bücher: vom Denken, vom Untersuchen und vom Erkennen, Leipzig, Hirzel, 1874.

—. «Le monde des idées », Philosophie, n. 91, 2006, 9-23.

Macnamara, J. A Border Dispute: The Place of Logic in Psychology, Cambridge MA, MIT Press, 1986.

Mander, W. J. An Introduction to Bradley's Metaphysics, Oxford, Clarendon Press, 1994.

- «Bosanquet and the Concrete Universal», The Modern Schoolman, vol. 77, 2000, 293-308.

—. "Bradley's Logic ", dans D. M. Gabbay \& J. Woods (dir.), Handbook of the History of Logic. Volume 4: British Logic in the Nineteenth Century, Amsterdam, Elsevier, 2008, 663-717.

Mansel, H. L., Prolegomena Logica: An Inquiry into the Psychological Character of Logical Processes, $2^{\mathrm{e}}$ édition, Boston, Gould \& Lincoln, 1860.

- The Philosophy of the Conditioned, London, Alexander Strahan, 1866.

Marion, M. "Oxford Realism: Knowledge and Perception ", British Journal for the History of Philosophy, vol. 8, 2000, 299-338 et 485-519. 
—. "L'épistémologie de Russell: de la logique mathématique aux vertus épistémiques », dans R. Nadeau (dir.), Philosophies de la connaissance. Contributions à une histoire de la théorie de la connaissance, Québec/Paris, Presses de l’Université Laval/Vrin, 2009, 281-316.

McDowell, J. «Anti-Realism and the Epistemology of Understanding », dans H. Parrett et J. Bouveresse (dir.) Meaning and Understanding, Berlin, Walter de Gruyter, 1981, 225-248.

Mill, J. S., An Examination of Sir William Hamilton's Philosophy, in Collected Works, vol. IX, Toronto, University of Toronto Press, 1979.

Moore, G. E. «La nature du jugement », dans Armengaud, 1985, 45-64. [1985a]

—. «La réfutation de l'idéalisme », dans Armengaud, 1985, 65-86. [1985b]

Moreland, J. P. Universals, Montréal et Kingston, McGill/Queen's University Press, 2001.

Passmore, John, A Hundred Years of Philosophy, Londres, Duckworth, 1957.

Philipse, H. «Psychologism and the Prescriptive Function of Logic », Grazer philosophische Studien, vol. 29, 1987, 13-33.

Popper, K. R., La logique de la découverte scientifique, Paris, Payot, 1973.

Prichard, H. A. "A Criticism of the Psychologists' Treatment of Knowledge ", Mind, n. s., vol. 16, 1907, 27-53.

Quine, W. v. "L'épistémologie devenue naturelle», dans Relativité de l'ontologie et autres essais, Paris, Aubier, 1977, 83-105.

Reid, T. The Works of Thomas Reid, W. Hamilton (dir.), $6^{\mathrm{e}}$ édition, Edimbourg, MacLachlan \& Stewart, volume 2, 1863.

Ribot, T. La Psychologie anglaise contemporaine. École expérimentale, $3^{\mathrm{e}}$ édition, Paris, Félix Alcan, 1914.

Robertson, G. C. "Association of Idea ", dans A. Bain et T. Whittaker (dir.) Philosophical Remains of George Croom Robertson, with a Memoir, Londres, Williams \& Norgate, 1894, 102-118.

—. "Association of Idea ", Encyclopaedia Britannica. XI $I^{\text {th }}$ Edition, 1910, vol. 2, 784-786.

Russell, B. Principles of Mathematics, Londres, Allen \& Unwin, 1903.

- Histoire de mes idées philosophiques, Paris, Gallimard, 1961.

—. Les problèmes de la philosophie, Paris, Payot, 1989.

—. Essais philosophiques, Paris, Presses Universitaires de France, 1997.

- Théorie de la connaissance. Le manuscrit de 1913, Paris, Vrin, 2002.

Ryle, G. "Introduction", dans A. J. Ayer, W. C. Kneale, G. A. Paul, D. F. Pears, P. F. Strawson, G. J. Warnock et R. A. Wollheim, The Revolution in Philosophy, Londres, MacMillan, 1956, 1-11.

Searle, J. Minds, Brains and Science, Cambridge MA, Harvard University Press, 1984.

Skorupski, J. "Mill on Language and Logic ", dans J. Skorupski (dir.), The Cambridge Companion to Mill, Cambridge, Cambridge University Press, 1998, 35-56.

Sprigge, T. L. S, James and Bradley. American Truth and British Reality, La Salle IL, Open Court, 1993.

Sober, E. "Psychologism ", Journal of the Theory of Social Behaviour, vol. 8, 1978, 165-191.

Simons, P. "L'intentionalité, la décennie décisive», dans D. Laurier \& F. Lepage (dir.), Essais sur le langage et l'intentionalité, Montréal/Paris, Bellarmin/Vrin, 1992, 17-33. 
Stern, R. "Hegel, British Idealism, and the Curious Case of the Concrete Universal », British Journal for the History of Philosophy, vol. 15, 2007, 115-153.

Stout, G. F., Analytic Psychology. 2 volumes, Londres, Swann Sonnenschein, 1896.

—. "Mr. Prichard's Criticism of Psychology", Mind, n. s., vol. 16, 1907, 236243.

—_. "Reply to Mr. Joseph », Mind, n. s., vol. 20, 1911, 11-14.

- "The Nature of Universals and Propositions ", réimprimé dans J. N. Findlay (dir.), Studies in Philosophy. British Academy Lectures, Londres, Oxford University Press, 1966, 5-24.

Stumpf, C., "Psychologie und Erkenntnistheorie», Abhandlungen der Königlich Bayerischen Akademie der Wissenschaften, vol. 19, $2^{\mathrm{e}}$ section, 1891, 465-516.

Twardowski, K. "Sur la théorie du contenu et de l'objet des représentations ", dans Husserl-Twardowski, Sur les objets intentionnels. 1893-1901, Paris, Vrin, 85200.

Ward, J. «Psychology», Encylopaedia Britannica. IX ${ }^{\text {th }}$ Edition, 1886, vol. 20, 37-85.

Warren, H. C. A History of Association Psychology, N.Y., Charles Scribner \& Sons, 1921.

Wilson, J. C. Statement and Inference, Oxford, Clarendon Press, 1926.

Wittgenstein, L. Recherches philosophiques, Paris, Gallimard, 2004.

Wollheim, R. F. H. Bradley, Harmondsworth, Penguin Books, 1959. 